Journal for ImmunoTherapy of Cancer

\title{
Use of pembrolizumab with or without pomalidomide in HIV-associated non-Hodgkin's lymphoma
}

Kathryn Lurain (D) , ${ }^{1}$ Ramya Ramaswami, ${ }^{1}$ Ralph Mangusan, ${ }^{1}$ Anaida Widell, ${ }^{1}$ Irene Ekwede, ${ }^{1}$ Jomy George, ${ }^{2}$ Richard Ambinder, ${ }^{3}$ Martin Cheever, ${ }^{4}$ James L Gulley (D) , ${ }^{5}$ Priscila H Goncalves, ${ }^{1}$ Hao-Wei Wang, ${ }^{6}$ Thomas S Uldrick (D) , ${ }^{1,7}$ Robert Yarchoan (D) ${ }^{1}$
To cite: Lurain $\mathrm{K}$,

Ramaswami R, Mangusan $R$, et al. Use of pembrolizumab with or without pomalidomide in HIV-associated non-Hodgkin's lymphoma. Journal for ImmunoTherapy of Cancer 2021;9:e002097. doi:10.1136/jitc-2020-002097

- Additional material is published online only. To view, please visit the journal online (http://dx.doi.org/10.1136/jitc2020-002097).

Accepted 13 January 2021
Check for updates

(C) Author(s) (or their employer(s)) 2021. Re-use permitted under CC BY-NC. No commercial re-use. See rights and permissions. Published by BMJ.

For numbered affiliations see end of article.

Correspondence to

Dr Kathryn Lurain;

kathryn.lurain@nih.gov

\section{ABSTRACT}

Background Non-Hodgkin's lymphoma (NHL) is currently the most common malignancy among people living with HIV (PLWH) in the USA. NHL in PLWH is more frequently associated with oncogenic viruses than NHL in immunocompetent individuals and is generally associated with increased PD-1 expression and T cell exhaustion. An effective immune-based second-line approach that is less immunosuppressive than chemotherapy may decrease infection risk, improve immune control of oncogenic viruses, and ultimately allow for better lymphoma control. Methods We conducted a retrospective study of patients with HIV-associated lymphomas treated with pembrolizumab \pm pomalidomide in the HIV and AIDS Malignancy Branch, Center for Cancer Research, National Cancer Institute.

Results We identified 10 patients with stage IV relapsed and/or primary refractory HIV-associated NHL who were treated with pembrolizumab, an immune checkpoint inihibitor, with or without pomalidomide. Five patients had primary effusion lymphoma (PEL): one had germinal center B cell-like (GCB) diffuse large B cell lymphoma (DLBCL); two had non-GCB DLBCL; one had aggressive B cell lymphoma, not otherwise specified; and one had plasmablastic lymphoma. Six patients received pembrolizumab alone at $200 \mathrm{mg}$ intravenously every 3 weeks, three received pembrolizumab $200 \mathrm{mg}$ intravenously every 4 weeks plus pomalidomide $4 \mathrm{mg}$ orally every day for days 1-21 of a 28-day cycle; and one sequentially received pembrolizumab alone and then pomalidomide alone. The response rate was $50 \%$ with particular benefit in gammaherpesvirus-associated tumors. The progression-free survival was 4.1 months (95\% Cl: 1.3 to 12.4 ) and overall survival was 14.7 months (95\% Cl: 2.96 to not reached). Three patients with PEL had leptomeningeal disease: one had a complete response and the other two had long-term disease control. There were four immune-related adverse events (irAEs), all CTCAEv5 grade 2-3; three of the four patients were able to continue receiving pembrolizumab. No irAEs occurred in patients receiving the combination of pembrolizumab and pomalidomide.

Conclusions Treatment of HIV-associated NHL with pembrolizumab with or without pomalidomide elicited responses in several subtypes of HIV-associated NHL. This approach is worth further study in PLWH and NHL.

\section{INTRODUCTION}

With current combination antiretroviral therapy (ART), the most common cancer among people living with HIV (PLWH) in the USA is non-Hodgkin's lymphoma (NHL). ${ }^{1}$ There is an 11-fold to 17-fold increased lifetime risk of NHL in PLWH compared with the general population, due in part to $\mathrm{CD} 4^{+}$ $\mathrm{T}$ cell lymphopenia but also to immune dysregulation, B cell activation, and immune exhaustion from chronic viral antigen stimulation. ${ }^{23}$ Similar to the general population, diffuse large B cell lymphoma (DLBCL) is the most common subtype, but there are also rare subtypes that occur almost exclusively in PLWH, such as primary effusion lymphoma (PEL) and plasmablastic lymphoma (PBL). In PLWH, DLBCL and PBL are frequently associated with Epstein-Barr virus (EBV). Kaposi sarcoma herpesvirus (KSHV, also known as human herpesvirus 8) is the etiologic agent of PEL, with EBV coinfection also present in the vast majority of cases. ${ }^{4}$ Several factors are believed to contribute to the increased risk of virus-associated NHL in PLWH. HIVassociated immunosuppression causes a loss of virus-specific $\mathrm{T}$ cells allowing decreased control of EBV-infected or KSHV-infected B cell, increasing $\mathrm{B}$ cell activation and the risk of malignant B cell transformation. ${ }^{5}$ Both EBV and KSHV are able to evade immune detection and maintain infection in host B cells through downregulation of expression of immune surface markers, which are essential for $\mathrm{T}$ cell and natural killer (NK) cell immune surveillance, and this process may work in tandem with HIV-induced immunosuppression. ${ }^{6}$

PD-1 inhibitors, such as pembrolizumab and nivolumab, have proven effective in many cancer subtypes, particularly those with neoantigens from a high mutational burden. ${ }^{7}$ 
Checkpoint inhibitors are potentially attractive agents in PLWH as they do not cause further immunosuppression, unlike traditional cytotoxic chemotherapies and radiotherapy, and also because of the increase in PD-1expressing exhausted T cells seen in PLWH compared with the general population. ${ }^{8}$ While these agents have had relatively disappointing results so far as single agents in aggressive NHL in the immunocompetent population, EBV-encoded and/or KSHV-encoded foreign antigens in HIV-associated NHL may render these tumors potentially more susceptible to such therapy. ${ }^{910}$ Recent clinical trials have provided evidence that these agents are safe in PLWH and have anti-tumor activity in a subset of cases. ${ }^{112}$

Pomalidomide is a third generation analog of thalidomide with immunomodulatory, antiangiogenic, and antineoplastic properties. Thalidomide and its analogs all work by binding to and modulating the activity of cereblon. Pomalidomide is approved by the United States Food and Drug Administration (FDA) for the treatment of multiple myeloma and Kaposi sarcoma, and has been proven to be safe in PLWH. ${ }^{13}$ Pomalidomide also has known activity in certain types of NHL. ${ }^{14}$ There may be direct anti-lymphoma activity in part via downregulation of IRF4/MUM1 expression, which is common in HIV-associated NHL. ${ }^{15} 16$ Pomalidomide can enhance T and NK cell activation, and there is also evidence that it may render EBV-infected or KSHV-infected tumor cells visible to the immune system by reversing EBV-induced and KSHV-induced downregulation of immune surface markers, such as MHC-1, ICAM-1, and CD86. ${ }^{17} 18$ Another clinically relevant aspect of pomalidomide is its ability to penetrate the central nervous system (CNS), as leptomeningeal involvement is more frequent in HIV-associated NHL. ${ }^{14} 19$

The standard treatment for NHL after progression on or after front-line chemotherapy is second-line combination chemotherapy, often containing a platinum agent, followed by autologous hematopoietic stem cell transplant. ${ }^{20}{ }^{21}$ This approach does not generally differ for HIV-associated NHL; however, a second-line approach that spares not only T cells, but also B cells and cells of the innate immune system may decrease infection risk, improve immune control of oncogenic viruses, and potentially allow for better outcomes. Given their potential activity in HIV-associated NHL and immune-cell sparing nature, checkpoint inhibitors and cereblon modulators may be beneficial for salvage use in patients with NHL in whom further immunosuppressive chemotherapy might negatively affect outcomes. Our group is involved in a clinical trial evaluating pembrolizumab in PLWH and cancer that included several patients with NHL and is conducting two clinical trials of pomalidomide in PLWH and Kaposi sarcoma (NCT02595866, NCT01495598, NCT02659930). These studies have provided evidence that these agents have similar safety profiles in PLWH as they do in the general population. ${ }^{11} 1322$ Based on this experience, we have treated additional patients with HIVassociated NHL with pembrolizumab with or without the addition of pomalidomide, and in this manuscript, we review our experience with this salvage therapy for HIVassociated NHL.

\section{PATIENTS AND METHODS}

\section{Study design and patient selection}

We conducted a retrospective study of all identified patients with HIV-associated lymphomas treated with pembrolizumab \pm pomalidomide in the HIV and AIDS Malignancy Branch, Center for Cancer Research, National Cancer Institute (NCI). All patients were participating in one or more clinical studies (NCT00006518, NCT00092222, NCT01419561) and one clinical trial (NCT02595866). All cases were pathologically confirmed in the Laboratory of Pathology, NCI, and evaluated at the time of active disease. Diagnoses were based on cytopathology and/or $\mathrm{H} \& \mathrm{E}$ staining of tissue supported by immunohistochemistry. Leptomeningeal involvement of NHL was determined by cytopathology and/or flow cytometry of the cerebrospinal fluid (CSF), and patients with CSF involvement were followed serially with cytopathology and flow cytometry. KSHV tumor status was confirmed by staining for latency-associated nuclear antigen (anti-ORF73 rat $\mathrm{mAB}$, Advanced Biotechnologies, Eldersburg, Maryland, USA). EBV tumor status was evaluated by in situ hybridization against EBV-encoded small RNA. Clinical records were reviewed for baseline clinical laboratory parameters prior to initiation of therapy. NHL staging and response to treatment were determined according to the Lugano classification for NHL, and restaging was performed every three cycles or more frequently if progressive disease was suspected. ${ }^{23}$ Disease stabilization of leptomeningeal disease was defined by the lack of new neurologic symptoms or MRI findings that could be attributed to disease. HIV viral loads and $\mathrm{CD} 4^{+}$and $\mathrm{CD} 8^{+} \mathrm{T}$ cell counts were generally measured every 3 months unless there was clinical indication to measure more frequently. Immunerelated adverse events (irAEs) were graded according to the Common Terminology Criteria for Adverse Events V.5.0 and managed according to the American Society of Clinical Oncological Clinical Practice Guidelines for management of irAEs. ${ }^{24} 25$

\section{Treatment}

Patients receiving pembrolizumab alone received $200 \mathrm{mg}$ intravenously every 3 weeks. Patients receiving both drugs received pembrolizumab $200 \mathrm{mg}$ intravenously every 4 weeks and pomalidomide $4 \mathrm{mg}$ orally every day for days 1-21 of a 28-day cycle, based on our experience developing pomalidomide for Kaposi sarcoma. ${ }^{13}$ The same dosing schedule of pomalidomide was used during periods when patients received pomalidomide as a single agent. Patients who developed grade 3 or 4 neutropenia had a dose reduction of pomalidomide to $3 \mathrm{mg}$ and received growth factor support with pegfilgrastim. All patients starting pomalidomide received thromboprophylaxis 
with either aspirin or enoxaparin. There were no changes in patients' ART prior to treatment initiation.

\section{Statistical considerations}

The data were censored on July 1, 2020. Response to treatment and immune reconstitution were evaluated using descriptive statistics. Progression-free survival (PFS) and overall survival (OS) were calculated from the time of initiation of pembrolizumab with or without the addition of pomalidomide until the date or progression, death or censor date as appropriate, and evaluated using KaplanMeier methodology.

\section{RESULTS}

\section{Patient characteristics}

Seven cisgender men and three cisgender women with stage IV HIV-associated NHL were treated with pembrolizumab \pm pomalidomide between June 2016 and October 2019 (table 1). They included three participants of Cancer Immunotherapy Trials Network 12 (NCT02595866) who have been included in a publication of the primary safety results of that study. ${ }^{11}$ There were a variety of lymphoma histologies among the treated patients: five had PEL; one had germinal center B cell like (GCB) DLBCL with $M Y C$ rearrangement; two had non-GCB DLBCL; one had aggressive B cell lymphoma, not otherwise specified (NOS); and one had PBL. In all, seven patients had gammaherpesvirus-associated NHL. All five PEL tumors were positive for KSHV and four were also positive for EBV. Also, one non-GCB DLBCL and the PBL were positive for EBV. The viral association could not be conclusively determined in the case of aggressive B cell lymphoma, NOS. Cytology and flow cytometry of the CSF was performed in all patients and revealed leptomeningeal involvement of NHL in four patients, three with PEL and one with EBV-negative non-GCB DLBCL. This included patient 6 with extracavitary PEL who was treated for asymptomatic CSF relapse only and had no evidence of systemic disease during treatment.

The median number of prior lines of therapies received prior to pembrolizumab \pm pomalidomide was 3 (range: $1-5)$. The most common therapy prior to pembrolizumab was dose-adjusted infusional etoposide, vincristine, and doxorubicin with cyclophosphamide and prednisone with or without rituximab (online supplemental table 1 ). The median time between the start of treatment with pembrolizumab and the last prior therapy was 56 days (range: 7-357). At baseline, median Eastern Cooperative Oncology Group performance score was 1 (range: 0-4) and median $\mathrm{CD}^{+}$and $\mathrm{CD}^{+} \mathrm{T}$ cell counts were 214 cells $/ \mu \mathrm{L}$ (range: $43-557$ ) and 607 cells $/ \mu \mathrm{L}$ (range: 126-1707), respectively. Four patients had a baseline CD4 count $<200$ cells $/ \mu \mathrm{L}$. All patients were receiving integrase inhibitor-based ART at the time of treatment initiation. ART in two patients included an HIV protease inhibitor (darunavir) boosted with ritonavir or cobicistat. The median HIV viral load at baseline was undetectable and no patient had a viral load $>400$ copies $/ \mathrm{mL}$ at baseline or at any time during treatment. No patient developed an opportunistic infection during treatment; however, one patient was receiving treatment at treatment initiation for extensive acyclovir-resistant genital herpes and CNS toxoplasmosis. One patient had concurrent hepatitis B infection and was receiving an ART regimen containing tenofovir and emtricitabine. Two patients had concurrent Kaposi sarcoma at treatment initiation.

\section{Treatment response and OS}

The median number of cycles of pembrolizumab received was 7 (range: 3-22); patient 7 was still receiving pembrolizumab and pomalidomide at the time of censor (table 2 and figure 1). Four patients also received pomalidomide: three received it concurrently with pembrolizumab, and patient 6 received it sequentially after pembrolizumab for leptomeningeal PEL. Pomalidomide was initiated in three patients (patients 6, 7, and 10) with PEL to treat leptomeningeal disease and in patient 9 with PBL when progressive disease was confirmed on pembrolizumab monotherapy.

Of the six patients who received pembrolizumab alone, there were three partial responses (PRs): one patient with PEL; one with aggressive B cell lymphoma, NOS; and one with EBV-positive non-GCB DLBCL (table 2 and figure 2). All PRs were observed at the first restaging, after three cycles of pembrolizumab. Patient 1 with PEL and patient 4 with aggressive B cell lymphoma, NOS with PRs did not experience subsequent disease progression on pembrolizumab and went on to receive allogeneic hematopoietic stem cell transplant and rituximab, ifosfamide, carboplatin, and etoposide followed by allogeneic hematopoietic stem cell transplant, respectively. Patient 5 with EBV-positive non-GCB DLBCL had a PR at the first restaging but then developed progressive disease after a total of six cycles of pembrolizumab. She went on to receive further treatment with rituximab, ibrutinib, and lenalidomide. Two patients with heavily pretreated lymphoma, patient 2 with $M Y C$ rearranged GCB DLBCL and patient 3 with PEL, had stable disease over 21 weeks and 18 weeks, respectively. Patient 8 with EBV-negative non-GCB DLBCL had progressive disease at first restaging.

Four patients received both pembrolizumab and pomalidomide (table 2). Two had received prior therapy with lenalidomide with subsequent progression of NHL. Patient 7 with refractory PEL had systemic and leptomeningeal complete response (CR) on pomalidomide and pembrolizumab and continued to receive these agents after CR to reduce risk of relapse. Patient 6 with leptomeningeal PEL had stable disease on pomalidomide monotherapy after pembrolizumab monotherapy. Patient 9 with PBL had rapidly progressive disease, and the addition of pomalidomide to pembrolizumab did not slow the rate of disease progression. Patient 10 with peritoneal and leptomeningeal PEL had a PR and significant improvement in performance status during treatment with pembrolizumab and pomalidomide. 


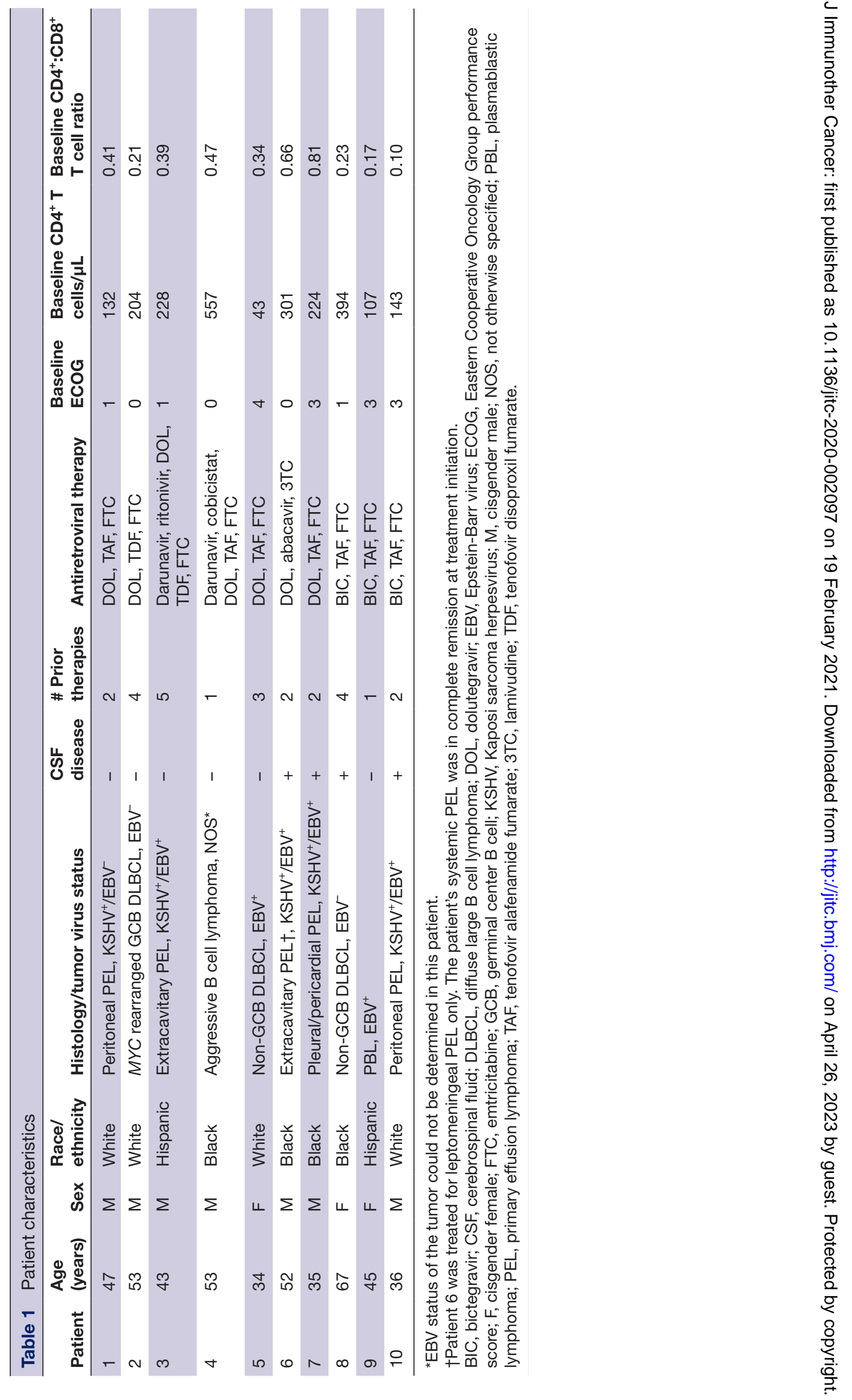




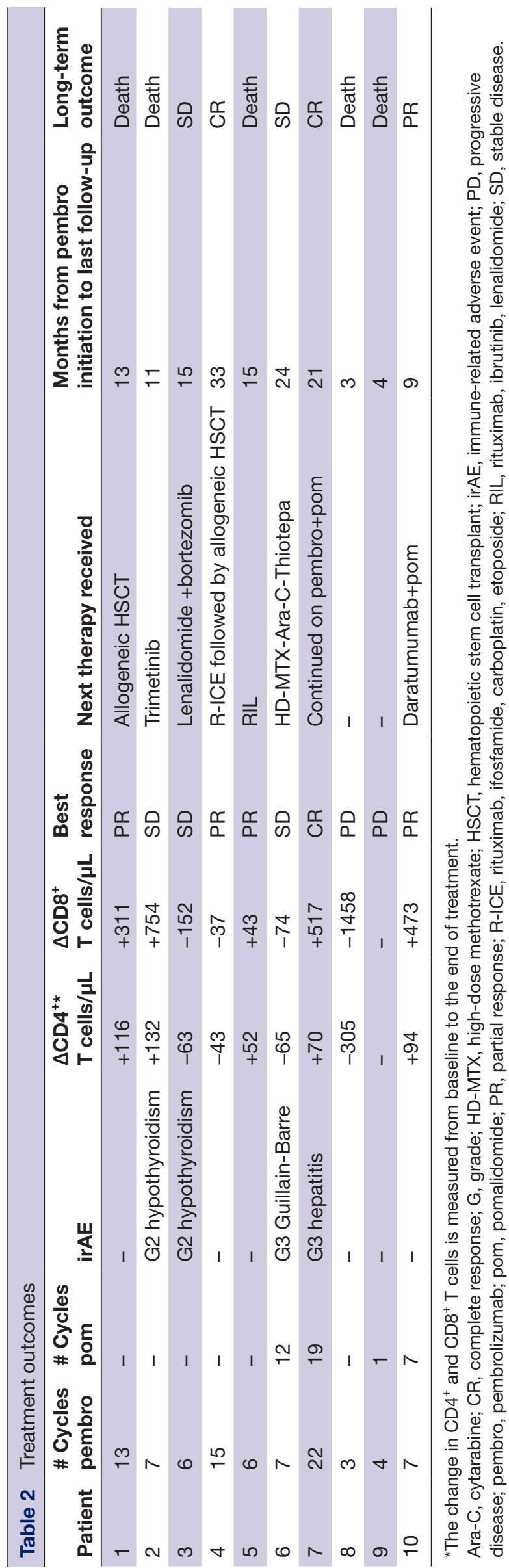

The overall response rate was $50 \%$ in all patients; four patients had a PR and one had a CR. The median PFS was 4.1 months (95\% CI: 1.3 to 12.4) and median OS was 14.7 months (95\% CI: 2.96 to not reached) (figure 3). The median time from initiation of pembrolizumab to last follow-up was 14 months (range: 3-33 months). Six of the seven patients with gammaherpesvirus positive tumors, including five with PEL, had CR, PR, or prolonged disease stabilization from treatment. The one patient (patient 9) with progressive disease as best tumor response had EBV-positive PBL. Of the two patients with tumors that were neither KSHV nor EBV positive (both DLBCL), patient 2 had disease stabilization for 21 weeks and patient 8 had progressive disease (tables 1 and 2). With regard to the effects on $\mathrm{T}$ cell numbers, lymphocyte counts were performed at end-of-treatment in nine patients. The median end-of-treatment $\mathrm{CD} 4^{+}$and $\mathrm{CD} 8^{+} \mathrm{T}$ cell counts were 237 and 634 cells $/ \mu \mathrm{L}$, respectively, which was a median increase of $52 \mathrm{CD}^{+}$and $43 \mathrm{CD}^{+} \mathrm{T}$ cells/ $\mu \mathrm{L}$ from baseline. Four patients experienced decreases in their $\mathrm{CD}^{+}$and/or $\mathrm{CD}^{+}$counts, but only two had decreases in $\mathrm{CD}^{+}$and/or $\mathrm{CD}^{+} \mathrm{T}$ cells of more than 100 cells $/ \mu \mathrm{L}$, which was attributed to tumor progression within the bone marrow.

If patients with PEL had evidence of leptomeningeal disease at baseline or during treatment, pomalidomide was administered with pembrolizimab or as a single agent after pembrolizumab. Out of the three patients treated for leptomeningeal PEL, one patient (patient 7) who had previously received extensive intrathecal chemotherapy for PEL in the CSF had a CR when pomalidomide was added to pembrolizumab. The second patient (patient 6) initially received pembrolizumab, but this had to be discontinued for an irAE (Guillain-Barre syndrome); he then received pomalidomide as a single agent and had disease stabilization for 12 months without subsequent irAEs. The third patient (patient 10) also had disease stabilization of his leptomeningeal PEL for more than 7 months on pembrolizumab and pomalidomide, and continued to receive pomalidomide for leptomeningeal disease as part of his treatment regimen after systemic progression of his PEL on pembrolizumab.

\section{Adverse events}

There were four irAEs (table 2). Two patients developed grade 2 hypothyroidism during cycle 4 of pembrolizumab and were treated with levothyroxine. One patient experienced grade 3 hepatitis during cycle 1 of pembrolizumab, which resolved with steroids, and treatment with pembrolizumab was reinitiated without further irAEs. Patient 6 developed grade 3 Guillain-Barre syndrome during cycle 7 of pembrolizumab, which resolved with a prolonged steroid taper and permanent cessation of pembrolizumab. This patient went onto receive pomalidomide without irAEs. No patient experienced an irAE while receiving pembrolizumab and pomalidomide together. 


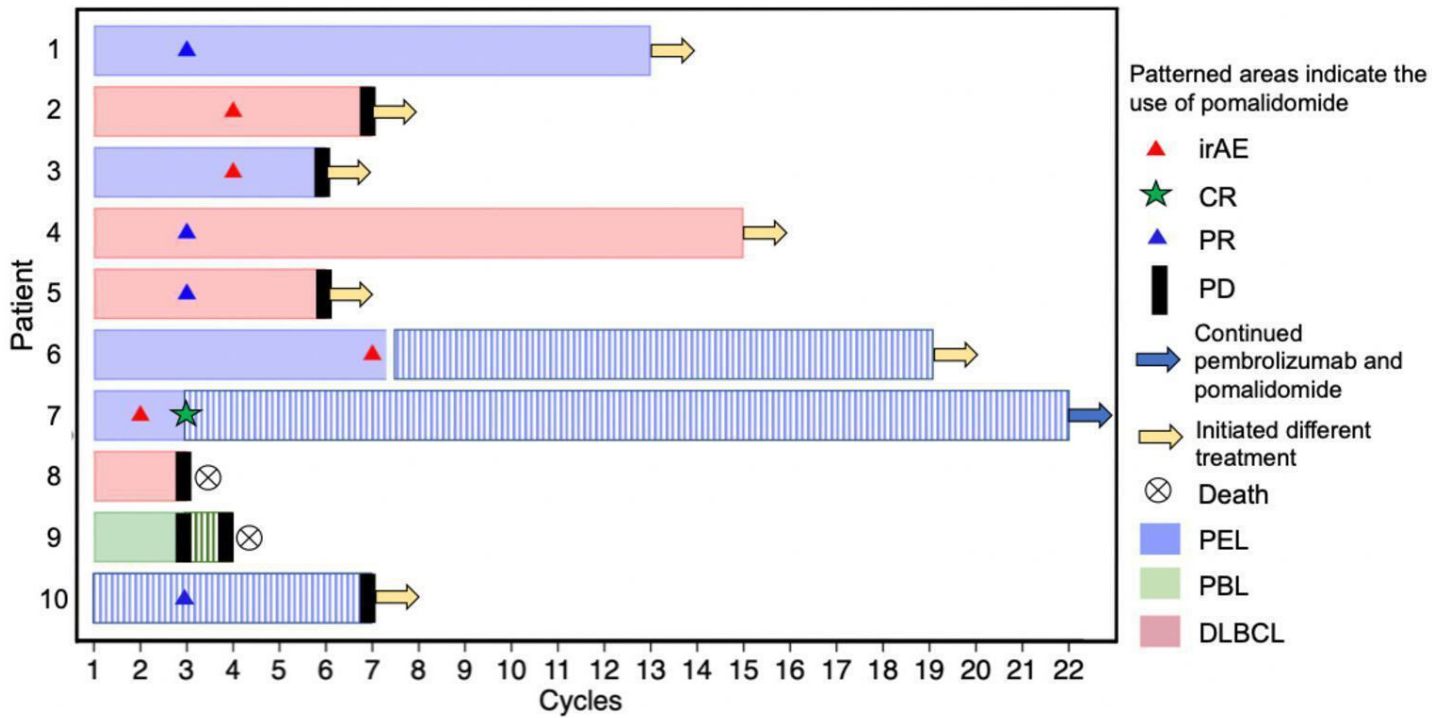

Figure $1 \mathrm{CR}$, complete response; DLBCL, diffuse large B cell lymphoma; irAE, immune-related adverse event; PBL, plasmablastic lymphoma; PD, progressive disease; PEL, primary effusion lymphoma; PR, partial response. *Patient 6 received pomalidomide sequentially after pembrolizumab was stopped due to an irAE

Two of the three patients with PEL receiving pomalidomide developed grade 3 neutropenia. In these cases, the dose of pomalidomide was reduced to $3 \mathrm{mg}$ and they received growth factor support with pegfilgrastim. Both patients continued to have intermittent grade 2 neutropenia but were able to continue treatment without infectious complications or episodes of febrile neutropenia.

\section{DISCUSSION}

This is one of the first reports to describe the use of pembrolizumab in HIV-associated NHL and the first to our knowledge to describe its use in combination with pomalidomide in this population. Although this is retrospective study of a small cohort of patients, the response rate of $50 \%$ indicates these agents should be investigated

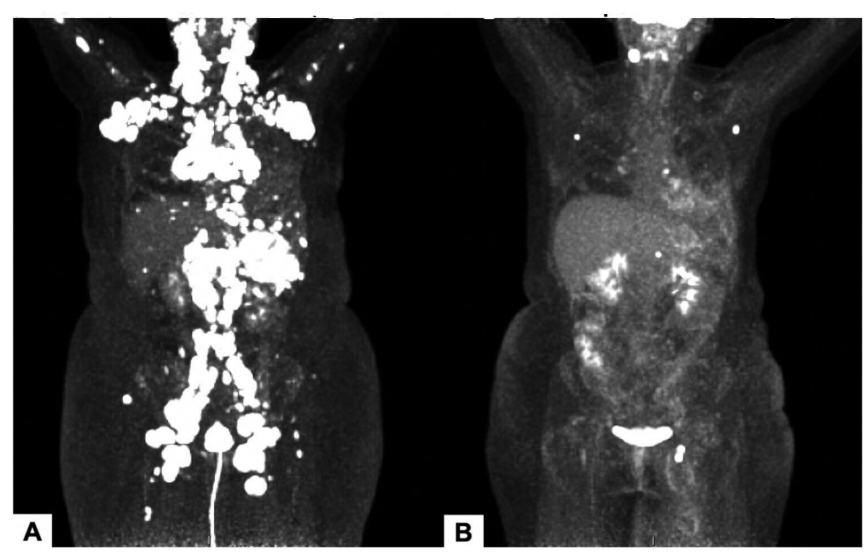

Figure $2{ }^{18} \mathrm{FDG}$ PET images of patient with $\mathrm{EBV}^{+}$diffuse large $B$ cell lymphoma (patient 5) prior to treatment (A) and after three cycles of pembrolizumab (B). EBV, Epstein-Barr virus; ${ }^{18} \mathrm{FDG}$ PET, fluorodeoxyglucose $\mathrm{F} 18$ positron emission tomography. further in HIV-associated NHL. Of particular note was the activity seen in PEL, a rare KSHV-driven lymphoma with an inferior OS compared with other HIV-associated NHLs. ${ }^{26}$ Of the five patients with PEL, one had CR with the combination of pembrolizumab and pomalidomide, two had PR with pembrolizumab alone, and two had disease stabilization with pembrolizumab over 18 and 21 weeks. Interestingly, the two patients with tumors negative for EBV and KSHV did not have responses to pembrolizumab, although one did have stable disease. DLBCL in the general population is usually EBV-negative and responses to single-agent checkpoint inhibitors are less than $10 \%$ in this population. ${ }^{10}$ Previous studies of HIV-uninfected patients have shown that the response to checkpoint inhibitor therapy may be higher in certain virus-assocated lymphomas, such as NK/T cell lymphomas. ${ }^{27} 28$ This may be due to the fact that virus-associated tumors express viral antigens and also tend to have higher expression of PD-1 and its ligand PD-L1, which has been associated with higher response to checkpoint inhibitor therapy. ${ }^{29} 30$ The results of this study suggests the agents should be studied further in gammaherpesvirus-associated NHL even outside the setting of HIV infection.

Also important to note was the activity of pomalidomide in patients with leptomeningeal involvement. Leptomeningeal PEL is a rare complication of an already rare lymphoma with only a handful of cases previously described in the literature. ${ }^{31}{ }^{32}$ To our knowledge this is the largest series of patients treated for leptomeningeal PEL and the first to describe the use of pomalidomide for treatment of PEL. The relatively high percentage of cases with leptomeningeal disease identified in this series suggests it may often go undiagnosed in patients with PEL. It is noteworthy that one of these patients attained a CR and the other two had long-term disease stabilization. This is a promising finding for the treatment of 


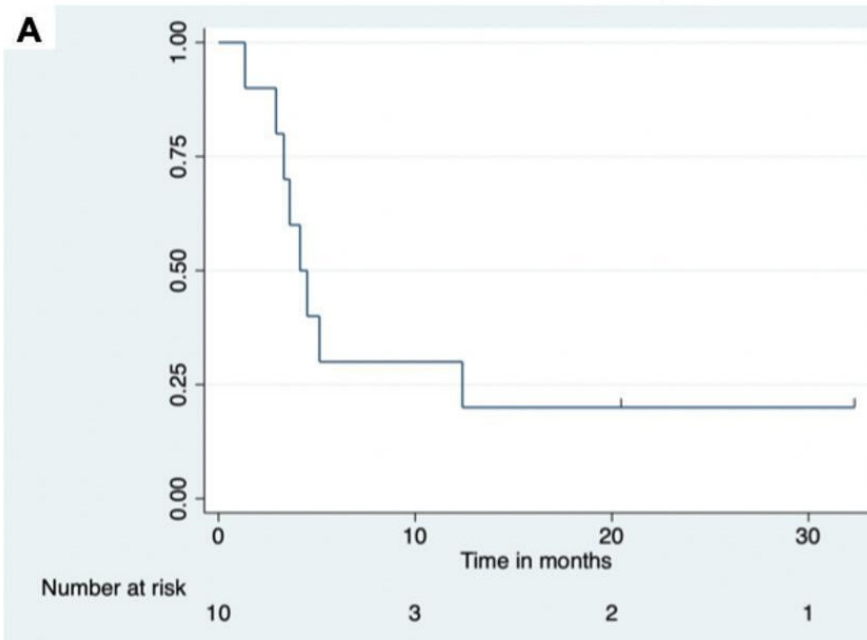

Figure 3 Progression-free survival (A) and overall survival (B).
B

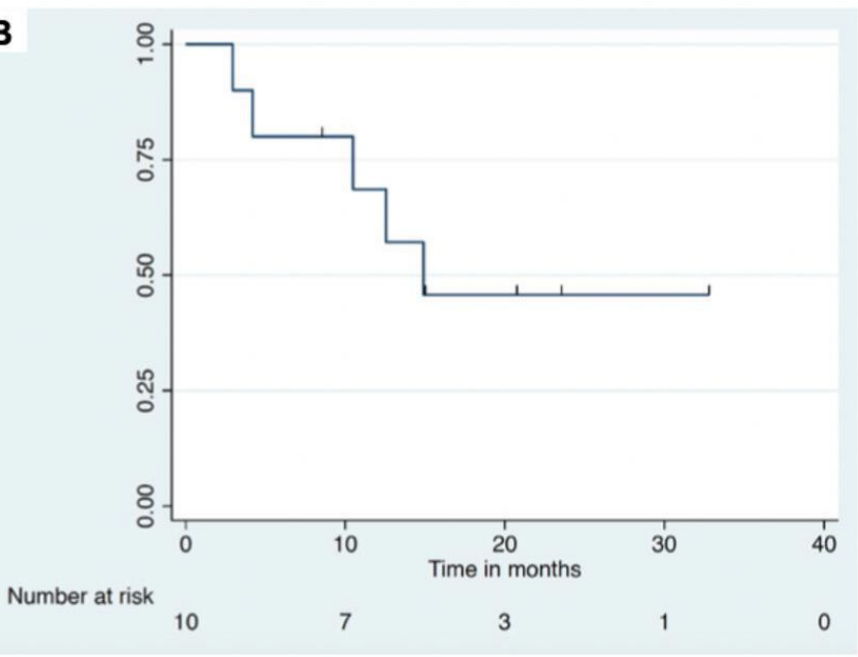

leptomeningeal NHL, particularly PEL, as this is notoriously difficult to treat and generally has an extremely poor prognosis. ${ }^{33} 34$

This study provides further evidence that checkpoint inibitors, such as pembrolizumab, are safe in PLWH and can be effective even in patients with relatively low $\mathrm{CD} 4^{+} \mathrm{T}$ cell counts (a PR was observed even in a patient with fewer than $50 \mathrm{CD} 4^{+} \mathrm{T}$ cells $/ \mu \mathrm{L}$ at baseline). Four patients on pembrolizumab monotherapy experienced irAEs, and all but one were able to continue with therapy. It also provides evidence that a checkpoint inhibitor can be safely administered with pomalidomide in this population. There have been safety concerns regarding the combination of PD-1 inhibitors and cereblon modulators (lenalidomide or pomalidomide) after randomized trials in relapsed multiple myeloma showed increased deaths in the combination arms compared with single-agent lenalidomide arms without a unifying cause for the increased deaths. ${ }^{35}$ An important distinction is that studies in multiple myeloma included dexamethasone along with checkpoint inhibitors, potentially negating the positive effects of anti-PD-1 therapy. Also, there is also no known single-agent benefit of anti-PD-1 therapy in multiple myeloma, unlike in certain subtypes of lymphoma. PLWH who develop cancer also tend to be appreciably younger than patients with multiple myeloma and have better organ function, particularly cardiovascular function, which appeared to be one source of AEs in the myeloma studies.

After front-line chemotherapy has failed, immune reconstitution and HIV control are vitally important for PLWH, not only for prevention of opportunistic infections but also for lymphoma control and OS. ${ }^{36}$ As is the case with immunocompetent patients with DLBCL, $\mathrm{CD} 4^{+}$lymphocytopenia post-chemotherapy among PLWH is associated with poorer survival, in part due to the increased risk of infection. ${ }^{37} 38$ In addition, aggressive lymphomas are immune-responsive tumors and ART-induced immune reconstitution plays an important role in tumor control. ${ }^{3940}$ Not only do checkpoint inhibitors allow for immune recovery, but the increase in PD-1 expression in circulating T cells in PLWH, even in the setting of well-controlled HIV with preserved $\mathrm{CD}^{+} \mathrm{T}$ cell counts, compared with the general population is an additional reason to consider checkpoint inhibitors in PLWH, potentially even to a greater extent than the immunocompetent population. ${ }^{84142}$ Cereblon modulators are also attractive agents for study in PLWH because they can induce T and NK cell activation and reverse immune surface marker downregulation by oncogenic viruses. ${ }^{43}$ There is also potential for synergy between checkpoint inhibitors and cereblon modulators, such as pomalidomide, and this will be evaluated in an upcoming clinical trial in our group for patients with virus-associated tumors in people with and without HIV. Most important, we hope these findings encourage investigators to include PLWH in prospective immunotherapy trials for NHL and other cancers per American Society of Clinical Oncology and FDA guidelines. ${ }^{445}$

\section{Author affiliations}

${ }^{1} \mathrm{HIV}$ and AIDS Malignancy Branch, Center for Cancer Research, National Cancer Institute, National Institutes of Health, Bethesda, Maryland, USA

${ }^{2}$ Clinical Pharmacokinetics Research Lab, Clinical Center Pharmacy, National Institutes of Health, Bethesda, Maryland, USA

${ }^{3}$ Department of Oncology, Johns Hopkins University School of Medicine, Baltimore, Maryland, USA

${ }^{4}$ Cancer Immunotherapy Trials Network, Fred Hutchinson Cancer Research Center, Seattle, Washington, USA

${ }^{5}$ Genitourinary Malignancies Branch, Center for Cancer Research, National Cancer Institute, National Institutes of Health, Bethesda, Maryland, USA

${ }^{6}$ Laboratory of Pathology, Center for Cancer Research, National Cancer Institute, National Institutes of Health, Bethesda, Maryland, USA

${ }^{7}$ Division of Global Oncology, Department of Medicine, Fred Hutchinson Cancer Research Center, Seattle, Washington, USA

Twitter James L Gulley @gulleyj1 and Thomas S Uldrick @ThomasUldrick

Acknowledgements We thank Kirsta Waldon for research and clinical care coordination as well as our patients and their families.

Contributors KL, RR, TSU, MC, JLG, and RY designed the study. KL, RR, RM, AW, IE, JG, PHG, RA, RY, and TSU cared for patients. H-WW performed flow cytometry and pathologic confirmation of leptomeningeal lymphoma. $\mathrm{KL}$ and $\mathrm{RR}$ collected and analyzed data. All authors contributed to writing and approving the manuscript. 
Funding This research was supported by the Intramural Research Program of the $\mathrm{NIH}$, National Cancer Institute and by US federal funds from the National Cancer Institute, National Institutes of Health, under contract no. HHSN261200800001E.

Competing interests Pembrolizumab for NCT02595866 was provided to the NCl by Merck and Co. KL, RR, TSU and RY report research funding through Cooperative Research and Development Agreements (CRADAs) between Celgene Corporation (now Bristol Myers Squibb, $\mathrm{Co}$ ) and the National Cancer Institute as well as a CRADA between EMD-Serrono and the National Cancer Institute. RY has also used drugs for his clinical or laboratory research provided to the NCI by Genentech Corp, EMD-Serono, Janssen Research, and CTI Biopharma. RY and TSU are co-inventors on US Patent 10,001,483 entitled 'Methods for the treatment of Kaposi's sarcoma or KSHV-induced lymphoma using immunomodulatory compounds, and uses of biomarkers'. The patent application for this was filed in part based on the results of $\mathrm{NCl}$ protocol 12-C-0047, entitled 'A Phase I/II Study of the Safety, Pharmacokinetics and Efficacy of Pomalidomide (CC-4047) in the Treatment of Kaposi Sarcoma in Individuals with or without HIV'. It is their understanding that foreign patents have also been filed for this invention. This invention was made as full-time employees of the US government under 45 Code of Federal Regulations Part 7. RY's spouse, who is also a US Government employee, has a patent on KSHV viral IL-6. All rights, title, and interest to these patents have been or should by law be assigned to the US Department of Health and Human Services. The government conveys a portion of the royalties it receives to its employee-inventors under the Federal Technology Transfer Act of 1986 (PL 99-502). TSU reports research funding to Fred Hutchinson Cancer Center from Roche and consulting fees $(<U S \$ 10,000)$ from AbbVie and Seattle Genetics. All other authors report no relevant conflicts of interest.

\section{Patient consent for publication Not required.}

Ethics approval The research was conducted under protocols approved by the Institutional Review Board of the National Cancer Institute and all patients gave written informed consent in accordance with the Declaration of Helsinki.

Provenance and peer review Not commissioned; externally peer reviewed.

Data availability statement The datasets used and/or analyzed during the current study are available from the corresponding author on reasonable request.

Supplemental material This content has been supplied by the author(s). It has not been vetted by BMJ Publishing Group Limited (BMJ) and may not have been peer-reviewed. Any opinions or recommendations discussed are solely those of the author(s) and are not endorsed by BMJ. BMJ disclaims all liability and responsibility arising from any reliance placed on the content. Where the content includes any translated material, BMJ does not warrant the accuracy and reliability of the translations (including but not limited to local regulations, clinical guidelines, terminology, drug names and drug dosages), and is not responsible for any error and/or omissions arising from translation and adaptation or otherwise.

Open access This is an open access article distributed in accordance with the Creative Commons Attribution Non Commercial (CC BY-NC 4.0) license, which permits others to distribute, remix, adapt, build upon this work non-commercially, and license their derivative works on different terms, provided the original work is properly cited, appropriate credit is given, any changes made indicated, and the use is non-commercial. See http://creativecommons.org/licenses/by-nc/4.0/

\section{ORCID iDs}

Kathryn Lurain http://orcid.org/0000-0002-5794-7292

James L Gulley http://orcid.org/0000-0002-6569-2912

Thomas S Uldrick http://orcid.org/0000-0001-6959-0924

Robert Yarchoan http://orcid.org/0000-0002-3057-1395

\section{REFERENCES}

1 Smith CJ, Ryom L, Weber R, et al. Trends in underlying causes of death in people with HIV from 1999 to 2011 (D:A:D): a multicohort collaboration. Lancet 2014;384:241-8.

2 Gibson TM, Morton LM, Shiels MS, et al. Risk of non-Hodgkin lymphoma subtypes in HIV-infected people during the HAART era: a population-based study. AIDS 2014;28:2313-8.

3 Fenwick C, Joo V, Jacquier P, et al. T-cell exhaustion in HIV infection. Immunol Rev 2019;292:149-63.

4 Carbone A, Cesarman E, Spina M, et al. HIV-associated lymphomas and gamma-herpesviruses. Blood 2009;113:1213-24.

5 Piriou E, van Dort K, Nanlohy NM, et al. Loss of EBNA1-specific memory CD4+ and CD8+ T cells in HIV-infected patients progressing to AIDS-related non-Hodgkin lymphoma. Blood 2005;106:3166-74.
6 Davis DA, Mishra S, Anagho HA, et al. Restoration of immune surface molecules in Kaposi sarcoma-associated herpes virus infected cells by lenalidomide and pomalidomide. Oncotarget 2017;8:50342-58.

7 Yarchoan M, Hopkins A, Jaffee EM. Tumor mutational burden and response rate to PD-1 inhibition. N Engl J Med 2017;377:2500-1.

8 Trautmann L, Janbazian L, Chomont N, et al. Upregulation of PD-1 expression on HIV-specific CD8+ T cells leads to reversible immune dysfunction. Nat Med 2006;12:1198-202.

9 Lesokhin AM, Ansell SM, Armand P, et al. Nivolumab in patients with relapsed or refractory hematologic malignancy: preliminary results of a phase lb study. J Clin Oncol 2016;34:2698-704.

10 Ansell SM, Minnema MC, Johnson P, et al. Nivolumab for relapsed/ refractory diffuse large B-cell lymphoma in patients ineligible for or having failed autologous transplantation: a single-arm, phase II study. $J$ Clin Oncol 2019;37:481-9.

11 Uldrick TS, Gonçalves PH, Abdul-Hay M, et al. Assessment of the safety of pembrolizumab in patients with HIV and advanced cancer-a phase 1 study. JAMA Oncol 2019;5:1332-9.

12 Gonzalez-Cao M, Morán T, Dalmau J, et al. Assessment of the feasibility and safety of Durvalumab for treatment of solid tumors in patients with HIV-1 infection: the phase 2 DURVAST study. JAMA Oncol 2020;6:1063-7.

13 Polizzotto MN, Uldrick TS, Wyvill KM, et al. Pomalidomide for symptomatic Kaposi's sarcoma in people with and without HIV infection: a phase I/II study. J Clin Oncol 2016;34:4125-31.

14 Tun HW, Johnston PB, DeAngelis LM, et al. Phase 1 study of pomalidomide and dexamethasone for relapsed/refractory primary CNS or vitreoretinal lymphoma. Blood 2018;132:2240-8.

15 Patil A, Manzano M, Gottwein E. CK1 $\alpha$ and IRF4 are essential and independent effectors of immunomodulatory drugs in primary effusion lymphoma. Blood 2018;132:577-86.

16 Gopalakrishnan R, Matta $\mathrm{H}$, Tolani B, et al. Immunomodulatory drugs target IKZF1-IRF4-MYC axis in primary effusion lymphoma in a cereblon-dependent manner and display synergistic cytotoxicity with BRD4 inhibitors. Oncogene 2016;35:1797-810.

17 Carbone A, Gloghini A, Larocca LM, et al. Expression profile of MUM1/IRF4, BCL-6, and CD138/syndecan-1 defines nove histogenetic subsets of human immunodeficiency virus-related lymphomas. Blood 2001;97:744-51.

18 Davis DA, Shrestha P, Aisabor Al, et al. Pomalidomide increases immune surface marker expression and immune recognition of oncovirus-infected cells. Oncoimmunology 2019;8:e1546544.

19 Noy A. Optimizing treatment of HIV-associated lymphoma. Blood 2019;134:1385-94.

20 Zelenetz AD, Gordon LI, Abramson JS, et al. NCCN guidelines insights: B-cell lymphomas, version 3.2019. J Natl Compr Canc Netw 2019;17:650-61.

21 Reid E, Suneja G, Ambinder RF, et al. Cancer in people living with HIV, version 1.2018, NCCN clinical practice guidelines in oncology. $J$ Natl Compr Canc Netw 2018;16:986-1017.

22 Ramaswami R, Lurain KA, Widell A, et al. A phase I trial of pomalidomide in combination with liposomal doxorubicin in patients with Kaposi sarcoma with or without other KSHV-associated diseases. Journal of Clinical Oncology 2020;38:11552.

23 Cheson BD, Ansell S, Schwartz L, et al. Refinement of the Lugano classification lymphoma response criteria in the era of immunomodulatory therapy. Blood 2016;128:2489-96.

24 Brahmer JR, Lacchetti C, Schneider BJ, et al. Management of immune-related adverse events in patients treated with immune checkpoint inhibitor therapy: American Society of clinical oncology clinical practice guideline. J Clin Oncol 2018;36:1714-68.

25 United States Department of Health and Human Services. Common terminology criteria for adverse events version 5.0, 2020. Available: https://ctep.cancer.gov/protocolDevelopment/electronic applications/docs/CTCAE_v5_Quick_Reference_5x7.pdf

26 Lurain K, Polizzotto MN, Aleman K, et al. Viral, immunologic, and clinical features of primary effusion lymphoma. Blood 2019;133:1753-61.

27 Li X, Cheng Y, Zhang M, et al. Activity of pembrolizumab in relapsed/ refractory NK/T-cell lymphoma. J Hematol Oncol 2018;11:15.

28 Kwong Y-L, Chan TSY, Tan D, et al. Pd1 blockade with pembrolizumab is highly effective in relapsed or refractory NK/T-cell lymphoma failing L-asparaginase. Blood 2017;129:2437-42.

29 Carbone A, Gloghini A, Carlo-Stella C. Are EBV-related and EBVunrelated Hodgkin lymphomas different with regard to susceptibility to checkpoint blockade? Blood 2018;132:17-22.

30 Laurent C, Fabiani B, Do C, et al. Immune-checkpoint expression in Epstein-Barr virus positive and negative plasmablastic lymphoma: a clinical and pathological study in 82 patients. Haematologica 2016;101:976-84. 
31 Jain S, Palekar A, Monaco SE, et al. Human immunodeficiency virusassociated primary effusion lymphoma: an exceedingly rare entity in cerebrospinal fluid. Cytojournal 2015;12:22.

32 Santonja C, Medina-Puente C, Serrano Del Castillo C, et al. Primary effusion lymphoma involving cerebrospinal fluid, deep cervical lymph nodes and adenoids. Report of a case supporting the lymphatic connection between brain and lymph nodes. Neuropathology 2017;37:249-58

33 Kuitunen $\mathrm{H}$, Kaprio E, Karihtala P, et al. Impact of central nervous system (CNS) prophylaxis on the incidence of CNS relapse in patients with high-risk diffuse large B cell/follicular grade 3B lymphoma. Ann Hematol 2020;99:1823-31.

34 Bernstein SH, Unger JM, Leblanc M, et al. Natural history of CNS relapse in patients with aggressive non-Hodgkin's lymphoma: a 20year follow-up analysis of SWOG 8516 -- the southwest Oncology Group. J Clin Oncol 2009;27:114-9.

35 Lesokhin AM, Bal S, Badros AZ. Lessons learned from checkpoint blockade targeting PD-1 in multiple myeloma. Cancer Immunol Res 2019;7:1224-9.

36 Gopal S, Patel MR, Yanik EL, et al. Association of early HIV viremia with mortality after HIV-associated lymphoma. AIDS 2013;27:2365-73.

37 Judd J, Dulaimi $\mathrm{E}$, Li T, et al. Low Level of Blood CD4 ${ }^{+} \mathrm{T}$ Cells Is an Independent Predictor of Inferior Progression-free Survival in Diffuse Large B-cell Lymphoma. Clin Lymphoma Myeloma Leuk 2017; 17:83-8.
38 Calkins KL, Chander G, Joshu CE, et al. Immune status and associated mortality after cancer treatment among individuals with HIV in the antiretroviral therapy era. JAMA Oncol 2020;6:227-35.

39 Lurain K, Uldrick TS, Ramaswami R, et al. Treatment of HIVassociated primary CNS lymphoma with antiretroviral therapy, rituximab, and high-dose methotrexate. Blood 2020;136:2229-32.

40 Oksenhendler E, Clauvel JP, Jouveshomme S, et al. Complete remission of a primary effusion lymphoma with antiretroviral therapy. Am J Hematol 1998;57:266.

41 Day CL, Kaufmann DE, Kiepiela P, et al. PD-1 expression on HIVspecific $T$ cells is associated with T-cell exhaustion and disease progression. Nature 2006;443:350-4.

42 Lurain K, Ramaswami R, Yarchoan R, et al. Anti-PD-1 and anti-PD-L1 monoclonal antibodies in people living with HIV and cancer. Curr HIVIAIDS Rep 2020;17:547-56.

43 Davis DA, Shrestha P, Aisabor Al, et al. Pomalidomide increases immune surface marker expression and immune recognition of oncovirus-infected cells. Oncoimmunology 2018;8:e1546544.

44 Uldrick TS, Ison G, Rudek MA, et al. Modernizing clinical trial eligibility criteria: recommendations of the American Society of clinical Oncology-Friends of cancer research HIV Working group. $J$ Clin Oncol 2017;35:3774-80.

45 Cancer clinical trial eligibility criteria: patients with HIV, hepatitis B virus or hepatitis $C$ virus infections: food and drug administration guidance for industry. Available: https://www.fda.gov/media/121319/ download [Accessed 1 September 2020]. 